\title{
SELETIVIDADE DE Lecanicillium lecanii (Zimm.) ZARE \& W. GAMS (CLASSE-FORMA: Hyphomycetes) AO PARASITÓIDE Trichogramma atopovirilia OATMAN \& PLATNER, 1983 (Hymenoptera: Trichogrammatidae)
}

\author{
Selectivity of Lecanicillium lecanii (Zimm.) Zare \& W. Gams (class-form: Hyphomycetes) \\ to the parasitoid Trichogramma atopovirilia Oatman \& Platner, 1983 \\ (Hymenoptera: Trichogrammatidae)
}

\author{
Leandro Pin Dalvi ${ }^{1}$, Ricardo Antônio Polanczyk², Dirceu Pratissoli ${ }^{3}$, Ricardo Lopes de Melo ${ }^{4}$, \\ Anderson Mathias Holtz
}

\begin{abstract}
RESUMO
Estudou-se no presente trabalho, as características biológicas de Trichogramma atopovirilia em ovos de Spodoptera frugiperda sob influência do fungo Lecanicillium lecanii, com o objetivo de verificar, em laboratório, se este entomopatógeno é seletivo ao parasitóide. Os estudos foram desenvolvidos no setor entomologia do Núcleo de Desenvolvimento Científico e Tecnológico em Manejo Fitossanitário "NUDEMAFI". Os indicativos de seletividade adotados foram: número de ovos parasitados, porcentagem de emergência (viabilidade), número de indivíduos por ovo, razão sexual e longevidade das fêmeas que parasitaram. Nenhum desses parâmetros foi afetado pelo fungo, levando à conclusão de que o mesmo é seletivo a T. atopovirilia, possibilitando ser conveniente a utilização simultânea de ambos os agentes de controle biológico no Manejo Integrado de Pragas.
\end{abstract}

Termos para indexação: Insecta, parasitóide, fungo entomopatogênico, MIP.

\begin{abstract}
The present work studied the biological characteristics of Trichogramma atopovirilia in eggs of Spodoptera frugiperda under influence of the fungus Lecanicillium lecanii, with the aim of verifing in laboratory if this entomopathogenic fungus is selective to the parasitoid. The studies were carried out in the entomology sector of Nucleus of Scientific \& Technological Development in Phytossanitary Management (NUDEMAFI). The indicatives of selectivity adapted used were: number of parasited eggs, emergency percentage (viability), number of individuals for egg, sex ratio and longevity of the females that parasited. None of these parameters were affected by the fungus, leading to the conclusion that the same is selective to $T$. atopovirilia, possibiliting the simultaneous utilization of both agents of biological control into the Integrated Management of Pests.
\end{abstract}

Index terms: Insecta, parasitoid, entomopathogenic fungus, IMP.

(Recebido em 10 de janeiro de 2007 e aprovado em 3 de maio de 2007)

\section{INTRODUÇÃO}

A partir do início da revolução verde, por volta da década de 50, ocorreram mudanças profundas na agricultura, caracterizadas principalmente pela disponibilidade de novas tecnologias, muitas delas baseadas na utilização extensiva e intensiva de produtos fitossanitários, causando grande redução na utilização do controle biológico (MOREIRA et al., 2002). No entanto, a popularização do uso de agroquímicos como forma única de controle de pragas culminou no seu uso indiscriminado causando muitos problemas, como a resistência de insetos e ácaros, ataques intensos de pragas tidas como secundárias, ressurgência de pragas, desequilíbrios biológicos e efeitos prejudiciais ao homem, insetos úteis e outros organismos, além de resíduos nos alimentos, água e solo (PARRA et al., 2002). Devido à necessidade de reverter esse quadro houve uma retomada na utilização de inimigos naturais, hoje normalmente inseridos em programas de Manejo Integrado de Pragas (MIP).

Em programas de MIP, táticas de segmentos diferentes de controle biológico podem ser utilizadas simultâneamente, principalmente em culturas de maior importância econômica e com alta diversidade de pragas. Assim, parasitóides do gênero Trichogramma quando

${ }^{1}$ Engenheiro Agrônomo, Mestrando em Produção Vegetal - Departamento de Produção Vegetal - Universidade Federal do Espírito Santo/UFES - Alto Universitário, s/no - Cx. P. 16 - 29500-000 - Alegre, ES - leandro-mpv@cca.ufes.br

${ }^{2}$ Professor Adjunto, Doutor em Entomologia - Departamento de Produção Vegetal - Universidade Federal do Espírito Santo/UFES - Alto Universitário, s/no - Cx. P. 16 - 29500-000 - Alegre, ES - rapolanc@yahoo.com.br

${ }^{3}$ Professor Adjunto, Doutor em Entomologia - Departamento de Produção Vegetal - Universidade Federal do Espírito Santo/UFES - Alto Universitário, s/no - Cx. P. 16 - 29500-000 - Alegre, ES - pratissoli@cca.ufes.br

${ }^{4}$ Mestre Entomologia - Departamento de Produção Vegetal - Universidade Federal do Espírito Santo/UFES - Alto Universitário, s/nº - Cx. P. 16 29500-000 - Alegre, ES - ricardolopesdemelo@yahoo.com.br

${ }^{5}$ Professor Adjunto, Doutor em Entomologia - Departamento de Produção Vegetal - Universidade Federal do Espírito Santo/UFES - Alto Universitário, s/no - Cx. P. 16 - 29500-000 - Alegre, ES - aholtz@insecta.ufv.br 
utilizados, podem ficar expostos a entomopatógenos, possibilitando a ocorrência de interações, tornando indispensável a avaliação prévia desses efeitos sobre os parasitóides (MAGALHÃES et al., 1998).

Beserra \& Parra (2004) destacam Trichogramma atopovirilia Oatman \& Platner (Hymenoptera: Trichogrammatidae) como a espécie que demonstra maior aceitação por ovos de $S$. frugiperda, a mais agressiva e com maior potencial para utilização no campo. Dentre os fungos entomopatogênicos, o fungo Lecanicillium lecanii (Classe-forma: Hyphomycetes) (Zimm.) Zare \& W. Gams, destaca-se por apresentar alta virulência contra uma gama de insetos hospedeiros, atuando em diversas culturas (ALVES, 1998).

Objetivou-se, aqui, estudar as características biológicas de Trichogramma atopovirilia (Hymenoptera: Trichogrammatidae) submetido ao parasitismo em ovos tratados com um formulado comercial à base de L. lecanii, visando determinar se o entomopatógeno é ou não seletivo ao parasitóide.

\section{MATERIAL E METODOS}

O trabalho foi realizado no setor de entomologia do Núcleo de Desenvolvimento Científico e Tecnológico em Manejo Fitossanitário "NUDEMAFI" situado no campus do Centro de Ciências Agrárias da UFES (CCA-UFES), Alegre-ES, em câmara climatizada, com temperatura de $25 \pm 1^{\circ} \mathrm{C}$, umidade relativa de $70 \pm 10 \%$ e fotofase de 14 horas.

Utilizaram-se, em cada tratamento, 20 fêmeas recém emergidas de Trichogramma atopovirilia, provenientes do biotério do NUDEMAFI, as quais foram individualizadas em tubos de vidro $(8,0 \times 2,5 \mathrm{~cm})$, tamponados com filme plástico de PVC contendo um filete de mel para alimentação. Cada fêmea foi considerada como uma repetição e recebeu uma cartela $(4,0 \times 2,0 \mathrm{~cm})$ de cartolina azul celeste, contendo 30 ovos do hospedeiro Spodoptera frugiperda (Lepidoptera: Noctuidae), coletados na criação estoque do NUDEMAFI, com no máximo 12 horas de idade. Esses ovos foram colados na cartolina com goma arábica a $10 \%$ e tratados por imersão durante cinco segundos, em água destilada (testemunha), ou em calda na concentração $10^{7}$ conídios $\mathrm{mL}^{-1}$ de bioinseticida à base de Lecanicillium lecanii $\left(\right.$ Vertirril $\left.^{\circledR}\right)$. As cartelas foram colocadas sobre papel de filtro, à temperatura ambiente, até a eliminação do excesso de água. Os ovos foram expostos ao parasitismo por 24 horas, após o que a cartela foi transferida para outro tubo, o qual permaneceu nas mesmas condições até a emergência dos descendentes. Os parâmetros indicativos de seletividade adotados foram: número de ovos parasitados, porcentagem de emergência (viabilidade), número de indivíduos por ovo, razão sexual e longevidade das fêmeas que parasitaram.

O delineamento experimental utilizado foi o inteiramente casualizado, as médias foram submetidas ao teste de Tukey, a $5 \%$.

\section{RESULTADOS E DISCUSSÃO}

A capacidade de parasitismo de T. atopovirilia não diferiu entre os tratamentos (Tabela 1), demonstrando que o potencial de parasitismo das fêmeas não foi afetado pelo fungo. Esses resultados foram superiores aos valores relatados por Morales et al. (2004), que avaliando o potencial biológico de fêmeas de T. atopovirilia em ovos de Sitotroga cerealella (Lepidoptera: Gelechiidae), obtiveram parasitismo próximo a 13 ovos em 24 horas, oferecendo a cada fêmea do parasitóide cartelas contendo 30 ovos da praga. Já Cañete \& Foerster (2003) oferecendo 50 ovos de Anticarsia gemmatalis (Lepidoptera, Noctuidae) relataram 30 ovos parasitados. Segundo Beserra \& Parra (2004) essas diferenças no parasitismo estão relacionadas à preferência pelo hospedeiro ou a maior fecundidade de Trichogramma em um determinado hospedeiro.

Ressalta-se que os resultados citados acima foram obtidos sem a adição de bioinseticidas. Broglio-Micheletti et al. (2006) testando a ação de alguns produtos fitossanitários para adultos de T. galloi mostram que a cepa $159 \mathrm{E}$ de Metarhizium anisopliae (Metschnicoff) Sorokin reduziu o parasitismo de ovos de Diatraea saccharalis (Lepidoptera: Crambidae) em 78,26\%, enquanto a cepa IPA 139E não diferiu da testemunha, mostrando assim que tanto a espécie, como a cepa do fungo podem influenciar no parasitismo.

A viabilidade de T. atopovirilia foi superior a $95 \%$ não havendo diferenças (Tabela 1). Através dos resultados obtidos observa-se que $L$. lecanii não teve influência negativa no desenvolvimento do parasitóide, o qual apresentou alta viabilidade. Os valores estão próximos aos encontrados por outros autores em hospedeiros diferentes. Beserra \& Parra (2004) relatam viabilidade acima de 93\%, nessa mesma espécie e hospedeiro. Nicoli et al. (2004), avaliando a viabilidade e a razão sexual de $T$. atopovirilia em ovos de Anagasta kuehniella (Lepidoptera: Pyralidae), obtiveram viabilidade de $96 \%$. No entanto para T. galloi, a aplicação da cepa 159E de $M$. anisopliae sobre ovos de $D$. saccharalis foi responsável por uma queda de $87,50 \%$ na viabilidade do parasitóide - (BROGLIO-MICHELETTI et al., 2006) - demonstrando que, em alguns casos, um entomopatógeno pode inviabilizar o embrião de Trichogramma. 
Quanto ao número de adultos emergidos por ovo, também não houve diferença entre os tratamentos (Tabela 1). Porém os resultados diferem dos apresentados por Beserra \& Parra (2004) que registraram 1,4 indivíduos por ovo de S. frugiperda. Segundo Bleicher \& Parra (1989) linhagens de Trichogramma de diferentes localidades podem apresentar variações biológicas e morfológicas afetando, por exemplo, o tamanho do parasitóide, o qual pode ser diretamente relacionado com o número de indivíduos por ovo.

A razão sexual não apresentou diferença entre os tratamentos (Tabela 1), apresentando valores acima de $80 \%$ de fêmeas em ambos tratamentos, sendo que, de acordo com Navarro (1998), o mínimo exigido é $50 \%$. Uma razão sexual alta mostra a boa adequação dos ovos do hospedeiro $S$. frugiperda ao parasitismo, embora também possa ser devido ao tempo de parasitismo, 24 horas, que corresponde à fase inicial da vida, pois, de acordo com Hoffmann et al. (1995), a razão sexual dos descendentes é inversamente proporcional ao tempo de vida da fêmea.

A longevidade de fêmeas que parasitaram também não diferiu (Tabela1), demonstrando que o entomopatógeno não teve efeito sobre a sobrevivência de T. atopovirilia. Os valores encontrados no presente estudo foram superiores aos de Cañete \& Foerster (2003) quando estudaram a biologia de T. atopovirilia, em ovos de Anticarsia gemmatalis (Lepidoptera, Noctuidae), com 6,6 dias para fêmeas nessas mesmas condições.

TABELA 1 - Valores médios \pm EP de taxa de parasitismo, viabilidade, indivíduos por ovo e razão sexual e longevidade de $T$. atopovirilia sobre ovos de $S$. frugiperda não tratados e tratados com L. lecanii (Vertirril) $\left(25 \pm 1^{\circ} \mathrm{C}, \mathrm{UR}=70 \pm 10 \%\right.$ e fotofase $\left.=14 \mathrm{~h}\right)$. Alegre, CCAUFES, 2006.

\begin{tabular}{lcc}
\hline Parâmetros & Testemunha & L. lecanii \\
\hline Ovos parasitados & $21,38 \pm 1,03 \mathrm{a}$ & $19,68 \pm 1,17 \mathrm{a}$ \\
Viabilidade \% & $95,30 \pm 0,95 \mathrm{a}$ & $97,85 \pm 0,73 \mathrm{a}$ \\
Indivíduos/ovo & $1,06 \pm 0,16 \mathrm{a}$ & $1,09 \pm 0,03 \mathrm{a}$ \\
Razão sexual & $0,81 \pm 0,09 \mathrm{a}$ & $0,81 \pm 0,05 \mathrm{a}$ \\
Longevidade/fêmeas & $8,00 \pm 0,47 \mathrm{a}$ & $8,05 \pm 0,40 \mathrm{a}$
\end{tabular}

Pares de médias seguidas pela mesma letra na linha não diferem pelo teste de Tukey a $\mathrm{p}<0,05$.

\section{CONCLUSÕES}

Em vista dos resultados pode-se concluir que $L$. lecanii é seletivo à $T$. atopovirilia, podendo-se utilizar esses dois agentes de controle biológico simultâneamente em programas de MIP.

\section{AGRADECIMENTOS}

Ao Conselho Nacional de Desenvolvimento Cientifico e Tecnológico $(\mathrm{CNPq})$ e à Coordenação de Aperfeiçoamento de Pessoal de Nível Superior (Capes), pela concessão de bolsas.

\section{REFERÊNCIAS BIBLIOGRÁFICAS}

ALVES, S. B. Controle microbiano de insetos. In: Fungos entomopatogênicos. Piracicaba: FEALQ, 1998. p. 289-382, $1163 \mathrm{p}$.

BESERRA, E. B.; PARRA, J. R. P. Biologia e parasitismo de Trichogramma atopovirilia Oatman \& Platner e Trichogramma pretiosum Riley (Hymenoptera, Trichogrammatidae) em ovos de Spodoptera frugiperda (J.E. Smith) (Lepidoptera, Noctuidae). Revista Brasileira de Entomologia, Londrina, v. 48, n. 1, p. 119-126, 2004.

BLEICHER, E.; PARRA, J. R. P. Espécies de Trichogramma parasitóides de Alabama argillacea: I. biologia de três populações. Pesquisa Agropecuária Brasileira, Brasília, v. 24 , n. 8, p. 929-940, 1989.

BROGLIO-MICHELETTI, S. M. F.; SANTOS, A. J. N.; PEREIRA-BARROS, J. L. Ação de alguns produtos fitossanitários para adultos de Trichogramma galloi Zucchi, 1988 (Hymenoptera: Trichogrammatidae). Ciência e Agrotecnologia, Lavras, v. 30, n. 6, p. 1051-1055, 2006.

CAÑETE, C. L.; FOERSTER, L. A. Incidência natural e biologia de Trichogramma atopovirilia Oatman \& Platner, 1983 (Hymenoptera, Trichogrammatidae) em ovos de Anticarsia gemmatalis Hübner, 1818 (Lepidoptera, Noctuidae). Revista Brasileira de Entomologia, Londrina, v. 47, n. 2, p. 201-204, 2003.

HOFFMANN, M. P.; WALKER, D. L.; SHELTON, A. M. Biology of Trichogramma ostriniae (Hym.: Trichogrammatidae) reared on Ostrinia nubilalis (Lep.: Pyralidade) and survey for additional hosts. Entomophaga, Paris, v. 40, n. 4, p. 387-402, 1995. 
MAGALHÃES, B. P.; MONNERAT, R.; ALVES, S. B. Interações entre entomopatógenos, parasitóides e predadores. In: Controle microbiano de insetos. Piracicaba: FEALQ, 1998. p. 195-216.

MORALES, J. S.; VÁSQUES, C.; GALLARDO, J. S.; GUTIERRES, F.; RÍOS, Y.; PÉREZ, N. L. Potencial biológico de Trichogramma Atopovirilia (Hymenoptera: Trichogrammatidae) como parasitóide de la plolilla de los granos. Bioagro, Barquisimeto-Cabudare, v. 16, n. 3, p. 197-204, 2004.

MOREIRA, J. C.; JACOB, S. C.; PERES, F.; LIMA, J. S.; MEYER, A.; OLIVEIRA-SILVA, J. J.; SARCINELLI, P. N.; BATISTA, D. F.; EGLER, M.; FARIA, M. V. C.; ARAÚJO, A. J.; KUBOTA, A. H.; SOARES, M. O.; ALVES, S. R.; MOURA, C. M.; CURI, R. Avaliação integrada do impacto do uso de agrotóxicos sobre a saúde humana em uma comunidade agrícola de Nova
Friburgo, RJ. Ciência \& Saúde Coletiva, São Paulo, v. 7, n. 2, p. 299-311, 2002.

NAVARRO, M. A. Trichogramma spp Procucción, uso y Manejo en Colombia. Guadalajara de Buga: Impretec, 1998. $176 \mathrm{p}$.

NICOLI, E. M.; PRATISSOLI, D.; REIS, E. F.; SANTOS, H. S. Viabilidade e razão sexual de Trichogramma atopovirilia Oatman \& Platner, 1983 (Hymenoptera, Trichogrammatidae) sob influência do hospedeiro Anagasta kuehniella (Zeller, 1879) (Lepidoptera, Pyralidae) em condições de laboratório. Entomologia y Vecterinaria, Rio de Janeiro, v. 11, n. 3, p. 521-533, 2004.

PARRA, J. R. P.; BOTELHO, P. S. M.; CORREAA-FERREIRA, B. S.; BENTO, J. M. S. Controle biológico: terminologia. In: Controle biológico no Brasil: parasitóides e predadores. São Paulo: Manole, 2002. p. 1-16. 